\title{
Pregnancy alters glucose-6-phosphate dehydrogenase trafficking, cell metabolism, and oxidant release of maternal neutrophils
}

\author{
Andrei L. Kindzelskii, ${ }^{1}$ Ji-Biao Huang, ${ }^{1}$ Tinnakorn Chaiworapongsa, ${ }^{2}$ Ryan M. Fahmy, ${ }^{1}$ \\ Yeon Mee Kim, ${ }^{2}$ Roberto Romero, ${ }^{2}$ and Howard R. Petty ${ }^{1}$ \\ ${ }^{1}$ Department of Biological Sciences, Wayne State University, Detroit, Michigan, USA \\ ${ }^{2}$ Perinatology Research Branch, National Institute of Child Health and Human Development, Hutzel Hospital, \\ Detroit, Michigan, USA
}

\begin{abstract}
Pregnancy is associated with changes in host susceptibility to infections and inflammatory disease. We hypothesize that metabolic enzyme trafficking affects maternal neutrophil activation. Specifically, immunofluorescence microscopy has shown that glucose-6-phosphate dehydrogenase (G-6-PDase), the rate-controlling step of the hexose monophosphate shunt (HMS), is located near the cell periphery in control neutrophils but is found near the microtubule-organizing centers in cells from pregnant women. Cytochemical studies confirmed that the distribution of the G-6-PDase antigen is coincident with functional G-6-PDase activity. Metabolic oscillations within activated pregnancy neutrophils are higher in amplitude, though lower in frequency, than activated control neutrophils, suggesting limited HMS activity. Analysis of radioisotope-labeled carbon flux from glucose to $\mathrm{CO}_{2}$ indicates that the HMS is intact in leukocytes from pregnant women, but its level is not enhanced by cell stimulation. Using extracellular fluorescent markers, activated pregnancy neutrophils were found to release reactive oxygen metabolites (ROMs) at a lower rate than activated control neutrophils. However, basal levels of ROM production in polarized pregnancy neutrophils were greater than in control neutrophils. Microtubule-disrupting agents reversed the observed changes in G-6-PDase trafficking, metabolic oscillations, and ROM production by maternal neutrophils. G-6-PDase trafficking appears to be one mechanism regulating ROM production by maternal neutrophils.
\end{abstract}

J. Clin. Invest. 110:1801-1811 (2002). doi:10.1172/JCI200215973.

\section{Introduction}

Survival of the fetal semi-allograft requires profound changes in the mother's host defense strategies. In addition to suppression of adaptive immune responses (1), changes in the functional properties of phagocytic cells have been noted. Reduced chemotaxis, adherence, reactive oxygen metabolite (ROM) release, phagocytosis, and microbial killing have been reported for neutrophils from pregnant women (2-8). The limited proinflammatory potential of leukocytes from pregnant women apparently renders them more susceptible to infections $(9,10)$. For example, in humans and/or animal models,

Received for publication May 21, 2002, and accepted in revised form November 5, 2002.

Address correspondence to: Howard R. Petty, Department of Biological Sciences, Wayne State University, Detroit, Michigan 48202, USA. Phone: (313) 577-2896; Fax: (313) 577-9008;

E-mail: hpetty@biology.biosci.wayne.edu.

Conflict of interest: The authors have declared that no conflict of interest exists.

Nonstandard abbreviations used: reactive oxygen metabolite (ROM); hexose monophosphate shunt (HMS); glucose-6phosphate (G-6-P); glucose-6-phosphate dehydrogenase (G-6-PDase); 6-aminonicotinamide (6-AN); phosphofructokinase (PFK); pyruvate kinase (PK); lactate dehydrogenase (LDH);

$N$-formyl-met-leu-phe (FMLP); resonance energy transfer (RET); dihydrotetramethylrosamine ( $\left.\mathrm{H}_{2} \mathrm{TMRos}\right)$; tetramethylrosamine (TMRos); microtubule-organizing center (MTOC). host resistance to Listeria monocytogenes, Toxoplasma gondii, Neisseria gonorrhoeae, and Plasmodium berghei is reduced during pregnancy (9-11). Increased incidence and/or severity of poliomyelitis, influenza, malaria, pneumonia, periodontal disease, septic shock, acute pyelonephritis, and other infectious diseases have also been reported to accompany pregnancy (9-12). Inasmuch as neutrophils play a key role in tissue damage (13), it may be postulated that their limited proinflammatory ability contributes to the remission of rheumatoid arthritis, multiple sclerosis, and uveitis during pregnancy (14-16). However, a rational mechanism underlying functional changes in maternal neutrophils has remained elusive.

An essential element of inflammatory responses is superoxide anion production (17). Superoxide and its downstream ROMs contribute to the destruction of invading pathogens and host tissue damage (16-19). Superoxide is produced by the NADPH oxidase according to the following: $\mathrm{NADPH}+2 \mathrm{O}_{2} \rightarrow \mathrm{NADP}^{+}+\mathrm{H}^{+}+2 \mathrm{O}_{2}^{-}$.

To elicit a robust response, transmembrane signals activate the NADPH oxidase (17) and supply it with NADPH. The primary source of NADPH is the hexose monophosphate shunt (HMS), whose activity is greatly increased by activation. NADPH production begins with enhanced glucose transport (18), a rate-controlling step in metabolism (20), which is necessary for superoxide production $(19,21,22)$. Heightened glucose transport is due to the 
coupling of glucose transport with hexokinase activity (21). During cell activation, hexokinase is translocated to the plasma membrane (23), where it accelerates glucose transport to provide glucose-6-phosphate (G-6-P) for glycolysis and the HMS (Figure 1). The HMS's first and ratecontrolling step is mediated by glucose-6-phosphate dehydrogenase (G-6-PDase), which converts G-6-P into 6-phosphogluconolactone with NADPH release. 6-Aminonicotinamide (6-AN), an inhibitor of G-6-PDase, inhibits ROM production but not metabolism (24). The HMS's second step is catalyzed by 6-phosphogluconate dehydrogenase, which yields NADPH and ribose 5-phosphate. Phosphofructokinase (PFK) is a key allosteric enzyme of glycolysis. Pyruvate kinase (PK) converts phosphoenolpyruvate to pyruvate. Lactate dehydrogenase (LDH) converts pyruvate to lactate. Only about $1-2 \%$ of the carbon flux enters the citric acid cycle in neutrophils (25). These enzymatic steps are generally unidirectional in living cells and, by definition, not at equilibrium. Moreover, these steps do not occur in isolation but rather form a complex network of chemical reactions regulated by cooperative interactions. These conditions promote the emergence of novel patterns of enzyme activity, such as temporal oscillations and spatial waves (26-29).

This study shows that G-6-PDase undergoes anterograde transport in neutrophils from nonpregnant women, but retrograde transport in cells from pregnant women. Microtubule-dependent retrograde transport of G-6-PDase apparently limits HMS activity and the extracellular release of ROMs; this mechanism may account for changes in disease susceptibility during pregnancy.

\section{Methods}

Patients. Peripheral blood samples were obtained from nonpregnant women and pregnant women with written informed consent, and the study was approved by the institutional review board. The nonpregnant group consisted of women in the secretory phase of the menstrual cycle who were not taking oral contraceptives and who had no history of acute or chronic inflammatory conditions. Women with normal pregnancies (from 20 weeks to term) had no medical or obstetric complications. Eligible patients were approached at the Detroit Medical Center (Detroit, Michigan, USA).

Cell preparation. Neutrophils were isolated using Ficoll-Hypaque (Sigma Chemical Co., St. Louis, Missouri, USA) density gradient centrifugation (30). Neutrophil viability was greater than $95 \%$ as assessed by trypan blue exclusion.

Reagents. Colchicine, vincristine, vinblastine, taxol, cytochalasin D, LPS (serotype 026:B6), $N$-formyl-met-leuphe (FMLP), 6-AN, PMA, and Brij-58 were obtained from Sigma Chemical Co. FITC and TRITC were obtained from Molecular Probes Inc. (Eugene, Oregon, USA). Rabbit anti-hexokinase, anti-G-6-PDase, and anti-PK polyclonal antibodies and a goat anti-LDH polyclonal antibody were obtained from Chemicon International Inc. (Temecula, California, USA). The anti-PFK antibody was the generous gift of R. Kemp (The Chicago Medical
School, North Chicago, Illinois, USA). Anti- $\boldsymbol{\gamma}$-tubulin and IFN- $\gamma$ were obtained from R\&D Systems Inc. (Minneapolis, Minnesota, USA). FITC- or TRITC-conjugated antibodies were prepared as described previously (30).

HMS activity. HMS activation was measured as described previously $(31,32)$. Cells $\left(\sim 2 \times 10^{6}\right)$ were incubated in a total volume of $0.8 \mathrm{ml}$ in media consisting of either $\left[\mathrm{D}-1-{ }^{14} \mathrm{C}\right]$-labeled or $\left[\mathrm{D}-6-{ }^{14} \mathrm{C}\right]$-labeled glucose (American Radiolabeled Chemicals Inc., St. Louis, Missouri, USA) at $0.5 \mu \mathrm{Ci} / \mathrm{ml}$ with $1 \mathrm{mM}$ glucose in PBS. The ${ }^{14} \mathrm{CO}_{2}$ produced was captured in a center well containing $0.5 \mathrm{ml}$ hyamine hydroxide (Research Products International Corp., Mount Prospect, Illinois, USA) and a strip of filter paper. Incubations were performed in sealed containers at $37^{\circ} \mathrm{C}$ in a shaking water bath for 4 hours. $\mathrm{CO}_{2}$ was released from the solution by addition of $1 \mathrm{ml}$ of $7 \mathrm{~N}$ trichloroacetic acid followed by incubation for 1 hour. Lastly, $3 \mathrm{ml}$ of scintillation fluid was added to the center well, followed by liquid scintillation counting.

G-6-PDase cytochemistry. G-6-PDase activity was localized within cells using cytochemical methods (33). Briefly, the G-6-PDase incubation medium consisted of phosphate buffer ( $\mathrm{pH} 7.4), 32 \%$ (wt/vol) polyvinyl alcohol, 5 mM G-6-P (VWR International, Batavia, Illinois, USA), $0.4 \mathrm{mM} \mathrm{NADP}{ }^{+}$(Calbiochem-Novabiochem Corp., San Diego, California, USA), $2.5 \mathrm{mM} \mathrm{MgCl}_{2}, 2.5$ $\mathrm{mM} \mathrm{NaN}_{3}, 0.16 \mathrm{mM}$ 1-methoxy-5-methyl-phenazinium methyl sulfate (Sigma Chemical Co.), and $2.5 \mathrm{mM}$ Nitro Blue Tetrazolium (VWR International). Cells were washed, fixed with $2 \%$ paraformaldehyde, incubated for 30 minutes, and then washed again. Samples were transilluminated using a 590 long-pass optical filter (Omega Optical Inc., Brattleboro, Vermont, USA) to enhance the reaction product's contrast.

Immunofluorescence staining. Neutrophils were placed on glass coverslips, incubated with reagents as described below, then fixed as described previously (23). Briefly, cells were fixed with $2 \%$ paraformaldehyde, permeabilized with $1 \%$ Brij-58, and fixed with $2 \%$ paraformaldehyde at room temperature for 20 minutes. Cells were washed with HBSS, labeled with antibodies at $4^{\circ} \mathrm{C}$ for 30 minutes, then washed with HBSS at room temperature.

Fluorescence microscopy. Cells were observed with an Axiovert fluorescence microscope (Carl Zeiss Inc., Thornwood, New York, USA) with mercury illumination interfaced to a computer using Scion image-processing software (NIH, Bethesda, Maryland, USA) (27). A narrow bandpass filter set (Omega Optical Inc.) was used with excitation at $485 / 22 \mathrm{~nm}$ and emission at $530 / 30 \mathrm{~nm}$ for FITC, and excitation at $540 / 20 \mathrm{~nm}$ and emission at $590 / 30 \mathrm{~nm}$ for TRITC. Long-pass dichroic mirrors of 510 and $560 \mathrm{~nm}$ were used for FITC and TRITC, respectively. For resonance energy transfer (RET) imaging, 485/22-nm and 590/30-nm optical filters were used for excitation and emission, respectively, in conjunction with a 510-nm dichroic mirror. The fluorescence images were collected with an IMax ICCD (Princeton Instruments Inc., Princeton, New Jersey, USA). RET was also examined using microspectrophotometry (28). 


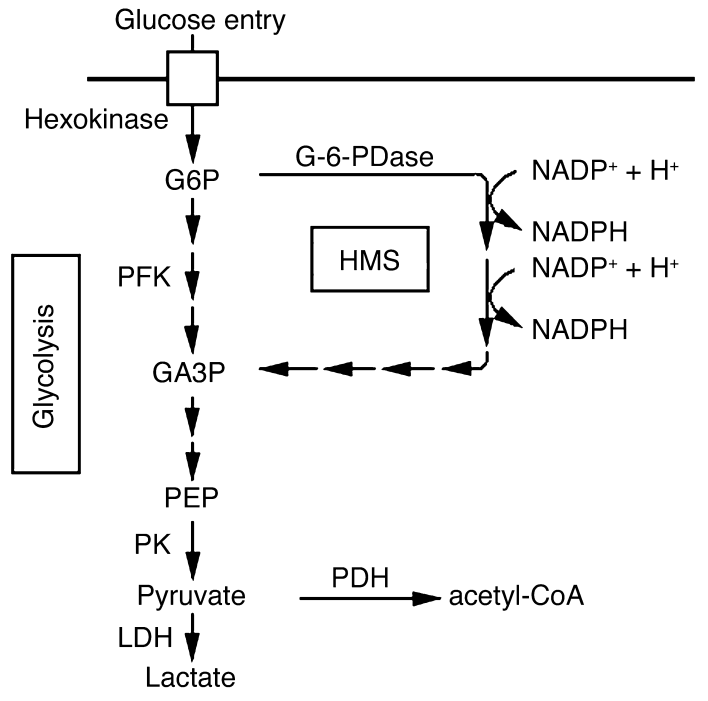

Figure 1

Glycolysis and the HMS. Enzyme activities are indicated by arrows. Important enzymes shown here are hexokinase, glucose-6-phosphate dehydrogenase (G-6-PDase), phosphofructokinase (PFK), pyruvate kinase (PK), and lactate dehydrogenase ( $\mathrm{LDH})$. The substrates listed here are: glucose-6-phosphate (G-6-P), phosphoenolpyruvate (PEP), and glyceraldehyde 3-phosphate (GA3P).

$N A D(P)$ H oscillations. $\mathrm{NAD}(\mathrm{P}) \mathrm{H}$ autofluorescence oscillations were detected as described previously $(30,34)$. An iris diaphragm was adjusted to exclude light from neighboring cells. A cooled photomultiplier tube held in a model D104 detection system (Photon Technology International Inc., Lawrenceville, New Jersey, USA) attached to a Carl Zeiss Inc. microscope was used $(30,34)$.

ROM production. Pericellular release of ROMs from single cells was monitored as described previously (35). Briefly, adherent neutrophils were surrounded in $2 \%$ gelatin containing $100 \mathrm{ng} / \mathrm{ml}$ dihydrotetramethylrosamine ( $\mathrm{H}_{2}$ TMRos) (Molecular Probes Inc.). ROMs, especially $\mathrm{H}_{2} \mathrm{O}_{2}$, released by the cells entered the gelatin matrix, where they oxidized $\mathrm{H}_{2}$ TMRos to tetramethylrosamine (TMRos), which was detected by fluorescence microscopy. The internal accumulation of ROMs was detected as described previously (36).

\section{Results}

Altered trafficking of G-6-PDase in neutrophils from pregnant women. Since G-6-PDase is the HMS's rate-controlling step, ROM release is decreased in activated cells from pregnant women $(2,3)$, and metabolic enzymes may undergo translocation during cell activation (23), we hypothesized that G-6-PDase trafficking is changed during pregnancy. To test this hypothesis, we examined the intracellular distribution of five metabolic enzymes in neutrophils from nonpregnant and pregnant women with and without in vitro stimulation with LPS. As illustrated in Figure 1, hexokinase, G-6-PDase, PFK, PK, and LDH are associated with multiple enzymatic steps from glucose phosphorylation through lactate formation. Untreated neutrophils, whether spherical or polarized in shape, show a uniform cytoplasmic distribution of hexokinase. When neutrophils were exposed to LPS (50 $\mathrm{ng} / \mathrm{ml}$ ) for 20 minutes, hexokinase was translocated from the cytoplasm to the cell periphery (Figure 2, a and b). This confirms previous work showing that hexokinase is translocated to plasma membranes during rat peritoneal macrophage activation with PMA (23). To determine whether hexokinase translocation was deficient in maternal neutrophils, we assessed the intracellular distribution of hexokinase in LPS-activated cells. Pregnancy neutrophils were competent to translocate hexokinase to the cell periphery (Figure 2, c and d). Hence, this cannot account for the reported changes in neutrophil function.

We next evaluated the distribution of G-6-PDase in neutrophils from nonpregnant and pregnant women under unstimulated and LPS-activated conditions (Figure 2, second row). We found that G-6-PDase is normally detected in the plasma membrane's vicinity in cells from nonpregnant women (Figure 2, e and $\mathrm{f}$ ). In contrast, G-6-PDase was not found near the plasma membrane in neutrophils from pregnant women (Figure 2, g and h). Quantitatively, cells from pregnant women demonstrated 3\% near the plasma membrane, $39 \%$ in the cytoplasm, and $58 \%$ as centrosomeassociated fluorescence. G-6-PDase translocation was observed in all of the samples from normal pregnant

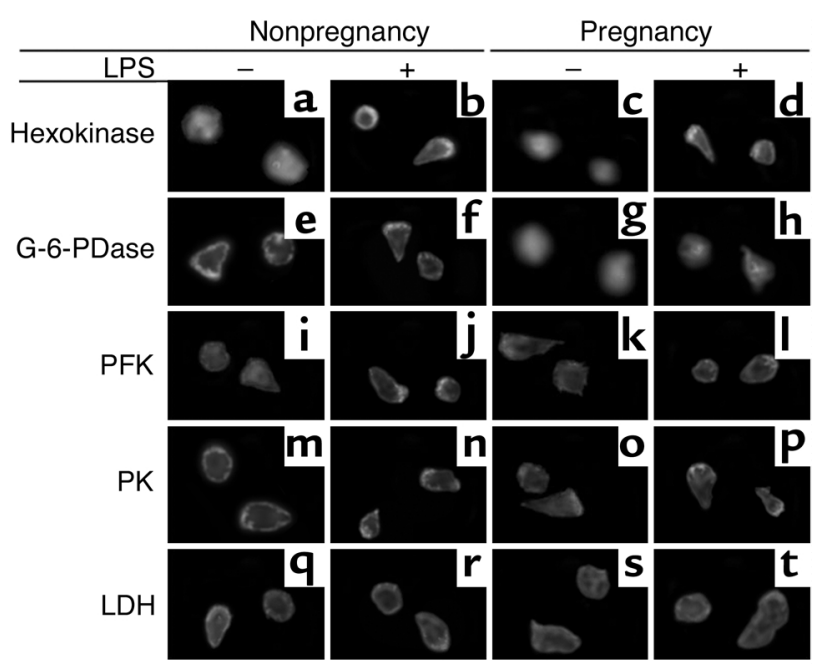

Figure 2

Immunofluorescence microscopy of metabolic enzymes. Representative neutrophils from nonpregnant women (first and second columns) and pregnant women (third and fourth columns) are shown. Neutrophils were stained with and without prior incubation with LPS ( $50 \mathrm{ng} / \mathrm{ml}, 20$ minutes). Cells were stained with antibodies directed against hexokinase (a-d), G-6-PDase (e-h), PFK (i-I), PK $(\mathbf{m}-\mathbf{p})$, and LDH $(\mathbf{q}-\mathbf{t})$. Hexokinase translocates to the periphery of stimulated neutrophils from nonpregnant and pregnant subjects (b and d). G-6-PDase is peripherally located under all conditions in cells from nonpregnant women but was found to be uniform or concentrated near the centrosome in maternal cells. Thus, G-6-PDase undergoes differential trafficking in cells from nonpregnant and pregnant women. Although PK undergoes significant trafficking in morphologically polarized neutrophils, no distinction between control and pregnancy neutrophils was noted. $n=16 . \times 480$. 

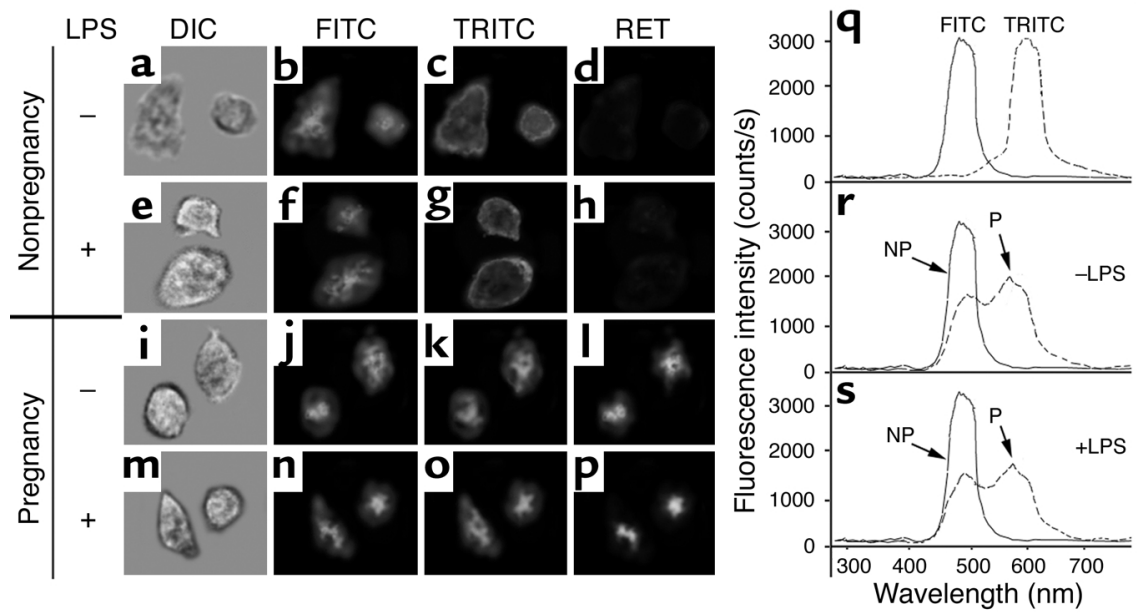

Figure 3

G-6-PDase accumulates near the centrosome in neutrophils from pregnant women. Cells from nonpregnant (NP) (a-h) and pregnant $(\mathrm{P})(\mathbf{i}-\mathbf{p})$ women were studied using direct immunofluorescence. Experiments were performed without $(\mathbf{a}-\mathbf{d}, \mathbf{i}-\mathbf{I}$, and $\mathbf{r})$ and with $(\mathbf{e}-\mathbf{h}, \mathbf{m}-\mathbf{p}$, and $\mathbf{s})$ prior incubation with LPS (50 ng/ml, 20 minutes). Cells were fixed, then stained with FITC-conjugated anti- $\gamma$-tubulin and TRITC-conjugated anti-G-6-PDase. Although $\gamma$-tubulin and G-6-PDase did not colocalize (b, c, f, and $\mathbf{g}$ ) or exhibit RET ( $\mathbf{d}$ and $\mathbf{h}$ ) in control neutrophils, both colocalization ( $\mathbf{j}, \mathbf{k}, \mathbf{n}$, and $\mathbf{o}$ ) and RET ( $\mathbf{I}$ and $\mathbf{p}$ ) were found in maternal neutrophils. RET was confirmed using fluorescence spectroscopy in $\mathbf{r}$ and $\mathbf{s}$. FITC and TRITC controls are shown in q. Thus, G-6-PDase accumulates near the centrosome of neutrophils from pregnant, but not nonpregnant, women. $n=9 . \times 550$.

women. Thus, neutrophils from pregnant women are characterized by a largely central distribution of G-6-PDase, in contrast to the peripheral distribution seen in $100 \%$ of the cells from nonpregnant individuals.

To ascertain the specificity of these changes in G-6-PDase distribution, we performed experiments using antibodies directed against other enzymes. We examined the intracellular distribution of PFK, PK, and LDH in nonpregnancy and pregnancy neutrophils with and without LPS stimulation (Figure 2, third through fifth rows). PFK was chosen because it is a primary allosteric regulator and is specific for the glycolytic pathway (Figure 1). Carbon flux through glycolysis and the HMS reaches $\mathrm{PK}$, whose activity is a rate-limiting step of glycolysis. Under the conditions tested, no major differ- ences between the distribution of these enzymes could be found in control and pregnancy cells. PK accumulated at the lamellipodium (Figure 2, fourth row). Interestingly, the three enzymes that can act as rate-controlling steps in glycolysis and/or the HMS accumulate at the cell periphery and lamellipodium. Similar results concerning the intracellular distributions of hexokinase, G-6-PDase, PFK, PK, and $\mathrm{LDH}$ were obtained using 50 nM FMLP (data not shown), which indicates that these changes in enzyme distribution are not specific for LPS. Furthermore, the activating stimulus PMA (30 nM for 30 minutes at $37^{\circ} \mathrm{C}$ ) had no effect on the distribution of G-6-PDase (data not shown), although some changes in metabolic oscillations and ROM production were noted (see below). Our experiments suggest that the trafficking of G-6-PDase is uniquely important in the regulation of neutrophil metabolism during pregnancy.

G-6-PDase traffics to the centrosome in cells from pregnant women. Figure 2 suggests that G-6-PDase undergoes retrograde motion in pregnancy neutrophils, in contrast to the anterograde distribution in cells from nonpregnant individuals. To test this possibility, cells were labeled with FITC-conjugated anti- $\gamma$-tubulin and TRITC-conjugated anti-G-6-PDase. $\gamma$-Tubulin is specific for microtubuleorganizing centers (MTOCs), including centrosomes of interphase cells, polar bodies of mitotic cells, and basal bodies of flagella. Figure 3 shows representative micrographs of neutrophils, from pregnant and nonpregnant women, that were stained with FITC/anti- $\gamma$-tubulin and TRITC/anti-G-6-PDase. As expected, anti- $\gamma$-tubulin

\section{Figure 4}

Colchicine reduces the pericentriolar distribution of G-6-PDase. LPS-stimulated cells from nonpregnant $(\mathbf{a}-\mathbf{i})$ and pregnant $(\mathbf{j}-\mathbf{r})$ women were studied using direct immunofluorescence in conjunction with microscopic imaging and spectrophotometry. Cells were fixed, then stained with FITC-conjugated anti- $\gamma$-tubulin and TRITC-conjugated antiG-6-PDase. The properties of $\gamma$-tubulin ( $\mathbf{a}, \mathbf{d}$, and g) and G-6-PDase (b,e, and $\mathbf{h}$ ) and RET (c, f, and i) between these labels were not significantly affected by colchicine or cytochalasin D in control neutrophils. However, colchicine (m-o) caused the G-6-PDase to become more uniformly distributed within pregnancy cells and reduced the amount of $\operatorname{RET}(\mathbf{o}) . n=5 . \times 560$.

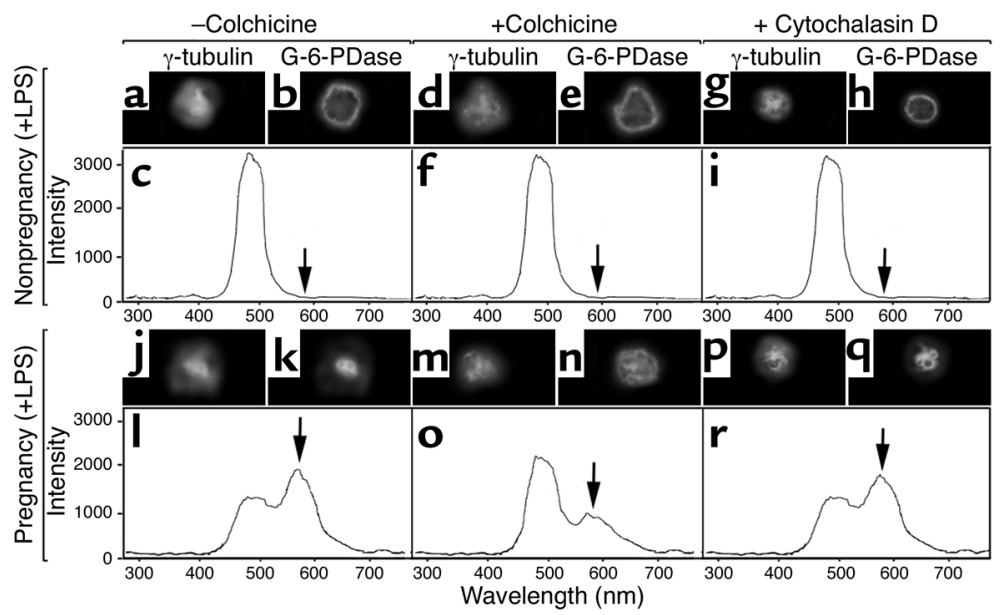




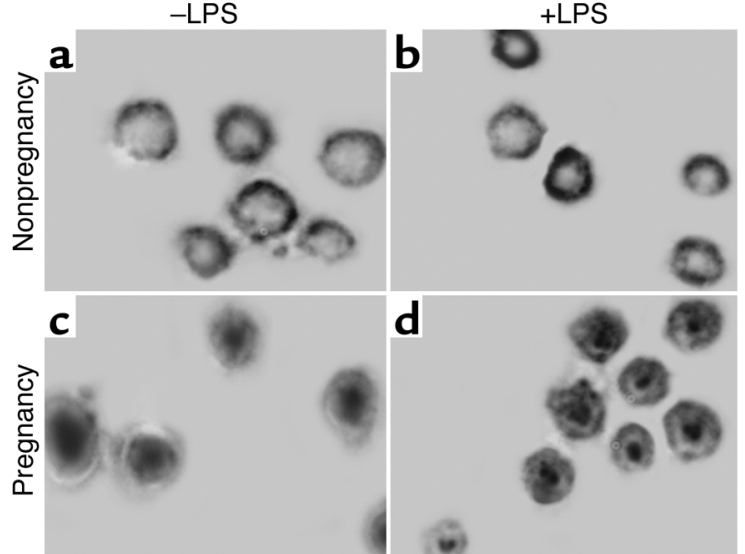

Figure 5

Localization of G-6-PDase activity in neutrophils. Representative cells from nonpregnant women ( $\mathbf{a}$ and $\mathbf{b}$ ) and pregnant women (c and $\mathbf{d}$ ) are shown. Cells were incubated in the absence ( $\mathbf{a}$ and $\mathbf{c}$ ) and presence (b and $\mathbf{d}$ ) of LPS ( $50 \mathrm{ng} / \mathrm{ml}, 20$ minutes). Cells were fixed and processed as described previously $(22,41)$. G-6-PDase activity is peripherally located in control cells ( $\mathbf{a}$ and $\mathbf{b}$ ) but was found to be enhanced near the centrosome in cells from pregnant women (c and d). $n=4 . \times 640$.

labeled the centrosome. As indicated above, neutrophils from nonpregnant women exhibited peripheral G-6-PDase labeling (Figure 3, $c$ and g). RET enables the detection of molecular proximity $(\leq 7 \mathrm{~nm})$ between two suitable fluorescent labels. Since anti-G-6-PDase is more abundant than $\gamma$-tubulin, we chose it as the acceptor label (TRITC), thereby optimizing RET. As expected, RET was not detected between these labels on neutrophils from nonpregnant women, using optical imaging (Figure $3 \mathrm{~d}$ ) or spectrophotometry (Figure 3r). Furthermore, LPS (50 $\mathrm{ng} / \mathrm{ml}$ for 20 minutes) had no effect on these parameters (Figure 3, h and s). When cells from pregnant women were stained with anti- $\gamma$-tubulin and anti-G-6-PDase, the FITC/anti- $\gamma$-tubulin and TRITC/ anti-G-6-PDase patterns overlapped substantially (Figure $3, j$ with $\mathrm{k}$ and $\mathrm{n}$ with $\mathrm{o}$ ). These results indicate that G-6-PDase traffics to the MTOC in maternal cells. However, since immunofluorescence does not demonstrate molecular proximity, we performed RET experiments. RET between these two labels was observed in the absence and presence of LPS, using microscopic imaging (Figure 3, 1 and $\mathrm{p}$ ) and spectrophotometry (Figure 3, $\mathrm{r}$ and s). Thus, G-6-PDase is translocated to the MTOC and is within approximately $7 \mathrm{~nm}$ of $\gamma$-tubulin in pregnancy, but not control, neutrophils.

G-6-PDase trafficking to the MTOC suggests that microtubules contribute to its intracellular localization. To examine this possibility, cells were treated with colchicine, which disrupts microtubules. Cells were treated with $50 \mu \mathrm{g} / \mathrm{ml}$ colchicine for 30 minutes at $37^{\circ} \mathrm{C}$ followed by LPS stimulation ( $50 \mathrm{ng} / \mathrm{ml}$ for 20 minutes). Although colchicine treatment had little effect on cells from nonpregnant women (Figure 4, d-f), colchicine changed the G-6-PDase distribution in cells from pregnant women (Figure 4, n vs. k). Furthermore, colchicine dramatically reduced RET between FITC/ anti- $\gamma$-tubulin and TRITC/anti-G-6-PDase (Figure 4, o vs. 1). These changes were not specific for colchicine, inasmuch as similar changes in G-6-PDase were noted after treatment of pregnancy cells with the microtubule-disrupting drugs vincristine $\left(5 \mu \mathrm{M}\right.$ for 40 minutes at $37^{\circ} \mathrm{C}$; $n=4)$ and vinblastine $\left(80 \mathrm{nM}\right.$ for 40 minutes at $37^{\circ} \mathrm{C}$; $n=3)$ (data not shown). However, the drug taxol $(30 \mu \mathrm{M}$ for 40 minutes at $37^{\circ} \mathrm{C}$ ), which stabilizes microtubules, had no effect on pregnancy neutrophils. To determine whether these changes were specific for microtubules, we tested the ability of the microfilament-disrupting drug cytochalasin D to influence the intracellular trafficking of G-6-PDase. Cytochalasin D was chosen over cytochalasin $\mathrm{B}$ as the latter can interact with the glucose transporter, thereby potentially altering metabolism via an alternate route. Neutrophils from nonpregnant and pregnant women were incubated with $50 \mu \mathrm{g} / \mathrm{ml}$ cytochalasin $\mathrm{D}$ for 30 minutes at $37^{\circ} \mathrm{C}$, conditions known to cause microfilament disassembly. In contrast to colchicine, cytochalasin $\mathrm{D}$ had no effect on the intracellular distribution of G-6-PDase in maternal neutrophils (Figure 4, $\mathrm{p}-\mathrm{r})$. Therefore, microtubules appear to play an important role in maintaining the intracellular distribution of G-6-PDase in cells from pregnant women.

Altered cytochemical localization of G-6-PDase activity in neutrophils from pregnant women. Our immunofluorescence microscopy experiments demonstrate a dramatic translocation of the G-6-PDase antigen in pregnancy neutrophils. To confirm the implicit assumption that distribution of G-6-PDase activity parallels its antigenic localization, we have employed cytochemical techniques to map G-6-PDase activity in cells (33). Figure 5 shows optical micrographs of neutrophils from pregnant and nonpregnant individuals, stained for G-6-PDase activity. In cells from nonpregnant women, G-6-PDase reaction product was predominantly found about a cell's periphery (Figure 5,

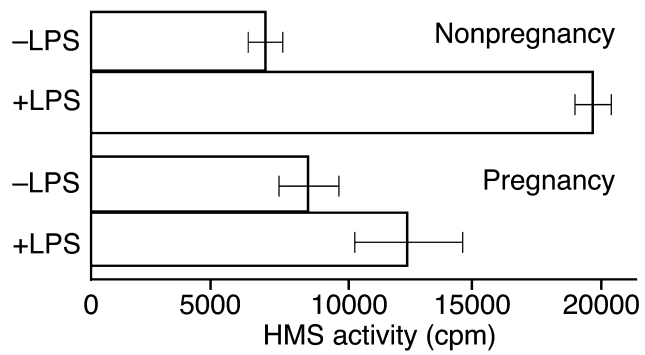

\section{Figure 6}

HMS activity in cells from nonpregnant and pregnant women with and without treatment with $50 \mathrm{ng} / \mathrm{ml} \mathrm{LPS}$. The metabolism of $1-{ }^{14} \mathrm{C}$ glucose and $6-{ }^{14} \mathrm{C}$-glucose to ${ }^{14} \mathrm{CO}_{2}$ was measured as described previously $(39,40)$. The $\mathrm{cpm}$ of ${ }^{14} \mathrm{CO}_{2}$ formed from $6-{ }^{-14} \mathrm{C}$-glucose was subtracted from the cpm of that formed from $1-{ }^{14} \mathrm{C}$-glucose. The cpm (HMS activity) is listed on the graph (mean \pm SEM). LPS substantially increases HMS activity in cells from nonpregnant individuals $(P<0.001$, paired two-tailed $t$ test $)$ but not from pregnant individuals $(P>0.01) \cdot n=7$. 


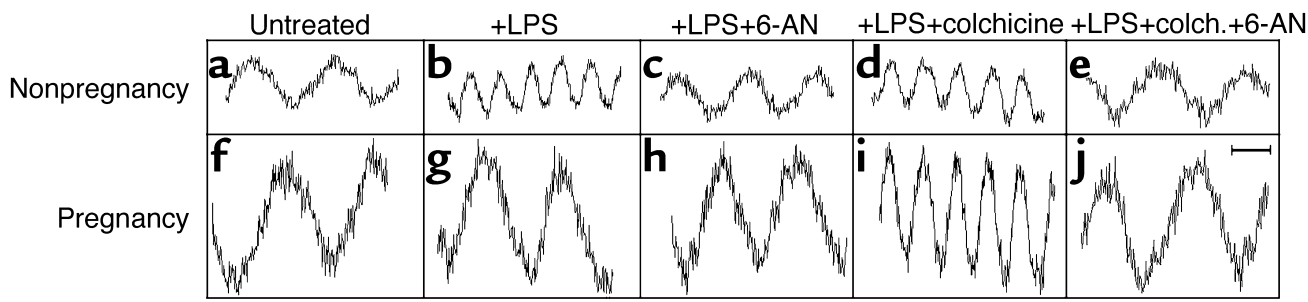

Figure 7

Representative $\mathrm{NAD}(\mathrm{P}) \mathrm{H}$ oscillations in adherent neutrophils from nonpregnant $(\mathbf{a}-\mathbf{e})$ and pregnant $(\mathbf{f}-\mathbf{j})$ women. Cells were untreated $(\mathbf{a}$ and f) or treated with LPS (b and $\mathbf{g}$ ), LPS plus 6-AN (c and $\mathbf{h})$, LPS plus colchicine (d and $\mathbf{i})$, or LPS plus colchicine and 6-AN (e and $\mathbf{j})$. The amplitudes of the $\mathrm{NAD}(\mathrm{P}) \mathrm{H}$ oscillations are higher in pregnancy cells (a and $\mathbf{f}$ ). LPS causes higher-frequency oscillations in cells from nonpregnant women (b), but not in cells from pregnant women $(\mathbf{g})$, which appears to be due to an inability to properly engage the HMS, as suggested by the inhibitor 6-AN ( $\mathbf{c}$ and $\mathbf{h}$ ). However, colchicine is able to restore the normal high-frequency oscillations to pregnancy cells (i) in a 6-AN-sensitive manner, suggesting that the ability to engage normal $\mathrm{NAD}(\mathrm{P}) \mathrm{H}$ flux has been restored. See Table 1 for quantitative results. Bar $=10$ seconds.

$a$ and $b$ ), as anticipated by the distribution of G-6-PDase antigen (Figure 2, e and f; Figure 3, $c$ and g; and Figure 4b). G-6-PDase activity remained in a peripheral distribution in cells incubated with $50 \mathrm{ng} / \mathrm{ml}$ LPS for $40 \mathrm{~min}-$ utes at $37^{\circ} \mathrm{C}$ (Figure 5 b). However, G-6-PDase activity was centrally located in cells from pregnant women (Figure 5, c and d) with or without treatment with LPS. These findings are consistent with the localization of G-6-PDase antigen (Figure 2, g and h; Figure 3, $\mathrm{k}$ and o; and Figure 4k). The staining patterns were not due to a nonspecific effect. Staining could not be observed during control experiments that omitted substrate (G-6-P and $\mathrm{NADP}^{+}$). Thus, both structural and functional assays indicate a dramatic reorganization of G-6-PDase in pregnancy neutrophils. These findings also illustrate that G-6-PDase remains active after translocation to the centrosomal region. In situ kinetic quantitative microphotometry studies indicate that the rates of product deposition in these experiments do not differ in maternal and control cells (data not shown). Thus,

Table 1

Quantitative analysis of metabolic oscillation properties of neutrophil populations from nonpregnant and pregnant women

Neutrophils from pregnant women

Treatment

$\begin{array}{lc} & \mathrm{HA} / \mathrm{HF} \\ \text { Untreated } & 0 \\ \text { LPS } & 12 \pm 4 \\ \text { PMA } & 0 \\ \text { Colchicine } & 0 \\ \text { 6-AN } & 0 \\ \text { IFN } & 0 \\ \text { LPS, 6-AN } & 1 \pm 1 \\ \text { LPS, colchicine } & 59 \pm 7 \\ \text { LPS, IFN } & 10 \pm 2 \\ \text { LPS, colchicine, 6-AN } & 0 \\ \text { Neutrophils from nonpregnant women } \\ \text { Treatment }\end{array}$

Treatment

Untreated

LPS

PMA

Colchicine

6-AN

IFN

LPS, 6-AN

LPS, colchicine

LPS, IFN

LPS, colchicine, 6-AN
$\mathrm{NAD}(\mathrm{P}) \mathrm{H}$ properties

$\begin{array}{ccc}\mathrm{HA} / \mathrm{LF} & \mathrm{LA} / \mathrm{HF} & \mathrm{LA} / \mathrm{LF} \\ 74 \pm 3 & 0 & 26 \pm 4 \\ 61 \pm 5 & 7 \pm 2 & 20 \pm 3 \\ 98 \pm 2 & 0 & 2 \pm 2 \\ 60 \pm 6 & 0 & 40 \pm 6 \\ 75 \pm 5 & 0 & 25 \pm 5 \\ 99 \pm 1 & 0 & 1 \pm 1 \\ 73 \pm 4 & 0 & 27 \pm 5 \\ 1 \pm 1 & 41 \pm 7 & 0 \\ 89 \pm 2 & 1 \pm 1 & 0 \\ 62 \pm 5 & 0 & 38 \pm 5\end{array}$

$\mathrm{NAD}(\mathrm{P}) \mathrm{H}$ properties

$\begin{array}{ccc}\text { HA/LF } & \text { LA/HF } & \text { LA/LF } \\ 0 & 0 & 100 \\ 0 & 98 \pm 2 & 2 \pm 2 \\ 99 \pm 1 & 0 & 1 \pm 1 \\ 0 & 0 & 100 \\ 0 & 0 & 100 \\ 99 \pm 1 & 0 & 1 \pm 1 \\ 0 & 2 \pm 1 & 98 \pm 1 \\ 0 & 100 & 0 \\ 0 & 1 \pm 1 & 0 \\ 0 & 0 & 100\end{array}$

ROM release properties

LA/LF

$\mathrm{HA} / \mathrm{HF}$
0
$13 \pm 5$
0
0
0
0
0
$60 \pm 6$
$10 \pm 3$
0

$\mathrm{HA} / \mathrm{LF}$
$73 \pm 4$
$60 \pm 5$
$97 \pm 2$
$60 \pm 7$
$75 \pm 6$
100
$74 \pm 5$
0
$90 \pm 3$
$62 \pm 6$

LA/HF
0
$8 \pm 3$
$1 \pm 1$
0
0
0
0
$40 \pm 6$
0
0

$27 \pm 4$

$18 \pm 3$

$2 \pm 1$

$40 \pm 7$

$25 \pm 6$

0

$26 \pm 5$

0

0

$38 \pm 6$

ROM release properties

$\mathrm{HA} / \mathrm{HF}$
0
0
0
0
0
0
0
0
100
0

$\mathrm{HA} / \mathrm{LF}$
0
0
$99 \pm 1$
0
0
$98 \pm 2$
0
0
0
0

$\mathrm{LA} / \mathrm{HF}$
0
$99 \pm 1$
0
0
0
0
$1 \pm 1$
100
0
$1 \pm 1$

LA/LF

100

$1 \pm 1$

$1 \pm 1$

100

100

$2 \pm 2$

$99 \pm 1$

0

0

$99 \pm 1$ $n$

16

16

3

16

12

11

12

16

11 12

The percentage of cells in each of the categories is shown. One hundred cells were measured for each treatment condition for each patient sample. The categories characterize the $\mathrm{NAD}(\mathrm{P}) \mathrm{H}$ oscillations or the time-dependence of ROM production as high amplitude/high frequency (HA/HF), high amplitude/low frequency (HA/LF), low amplitude/high frequency (LA/HF), and low amplitude/low frequency (LA/LF). 


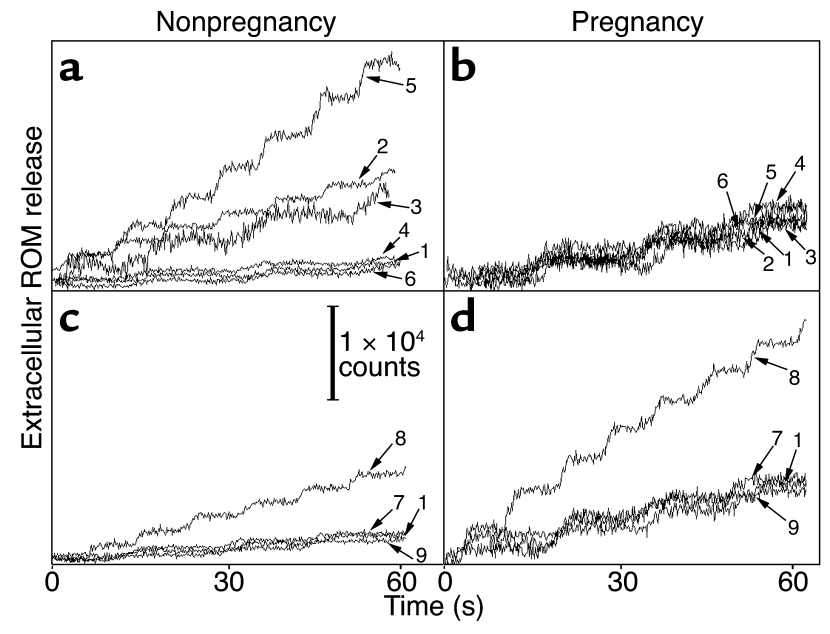

Figure 8

Rates of ROM release by neutrophils from nonpregnant (a and $\mathbf{c}$ ) and pregnant subjects (b and $\mathbf{d}$ ). ROM release was measured during cell migration through a matrix containing $\mathrm{H}_{2}$ TMRos (35). For all panels, the cells are adherent and traces labeled 1-9 are: 1, untreated; 2, LPS; 3, IFN- $\gamma$; 4, 6-AN; 5, IFN- $\gamma$ plus LPS; 6 , LPS plus 6 -AN; 7 , colchicine; 8, LPS plus colchicine; and 9, LPS plus colchicine and 6-AN. The greatest rates of ROM release from pregnancy neutrophils require the addition of colchicine. See Table 1 for further quantitative results. Vertical bar $=10,000$ counts.

G-6-PDase remains functional during pregnancy, but its activity is localized to the centrosome.

Effect of pregnancy on HMS activity. Although the cytochemical findings clearly demonstrate the location of G-6-PDase activity in neutrophils, this does not necessarily reflect the HMS's activity in living cells. To ascertain HMS activity, we measured the formation of ${ }^{14} \mathrm{CO}_{2}$ from $1-{ }^{14} \mathrm{C}$-glucose and $6-{ }^{14} \mathrm{C}$-glucose in the presence and absence of $50 \mathrm{ng} / \mathrm{ml} \mathrm{LPS} \mathrm{in} \mathrm{control} \mathrm{and} \mathrm{maternal}$ cells. Subtracting the amount of ${ }^{14} \mathrm{CO}_{2}$ formed during incubation with $6{ }^{-14} \mathrm{C}$-glucose from that formed in parallel experiments from $1{ }^{14} \mathrm{C}$-glucose provides an indication of HMS activity (31). Figure 6 shows the HMS activity in leukocytes from pregnant and nonpregnant women in the presence and absence of LPS $(50 \mathrm{ng} / \mathrm{ml})$. A substantial level of HMS activity is present in unstimulated cells from pregnant and nonpregnant women. As previously reported by others (31), cells from nonpregnant individuals undergo a dramatic increase in HMS activity in response to stimuli activating the respiratory burst (Figure 6). However, no significant change in HMS activity was noted in samples from pregnant women incubated with LPS (Figure 6). Thus, unstimulated neutrophils from pregnant women possess HMS activity, but this activity is not upregulated by LPS.

Effect of pregnancy on oscillatory metabolic flux in neutrophils. Previous studies have shown that neutrophil metabolism oscillates in time and that these oscillations correlate with cell functions, including ROM production (26). Therefore, we sought to provide direct evidence that the dynamics of cell metabolism is affected during pregnancy. To do this, we measured the time dependence of
$\mathrm{NAD}(\mathrm{P}) \mathrm{H}$ production, as judged by $\mathrm{NAD}(\mathrm{P}) \mathrm{H}$ autofluorescence, in adherent neutrophils. As previously reported $(26,29)$, polarized control neutrophils exhibit $\mathrm{NAD}(\mathrm{P}) \mathrm{H}$ oscillations with a period of about 20 seconds (Figure 7a). LPS stimulation decreases the oscillatory period to about 10 seconds (Figure 7b). 6-AN, an inhibitor of G-6-PDase (24), blocks the LPS-mediated enhancement of $\mathrm{NAD}(\mathrm{P}) \mathrm{H}$ oscillations (Figure 7c). This suggests that the 10-second metabolic period is associated with HMS activation. However, colchicine had no effect on the metabolic flux in control neutrophils (Figure $7 \mathrm{~d}$ ). In contrast to the effects of LPS, PMA treatment increased the amplitude of metabolic oscillations (Table 1). Thus, priming and/or activating stimuli can affect metabolism via multiple pathways.

When cells from normal pregnancy were examined, they demonstrated oscillations with a 20 -second period, as did those from nonpregnant women. However, in contrast to control cells, about $70 \%$ of the pregnancy neutrophils demonstrated high-amplitude oscillations (Table 1), as if they had been primed by IFN- $\gamma$ (34). Moreover, LPS and 6-AN had no effect on maternal neutrophils (Figure 7, f-h). However, PMA treatment did increase the proportion of pregnancy cells that exhibited high-amplitude oscillations from $74 \%$ to $98 \%$ (Table 1). When neutrophils of pregnant women were treated with colchicine $\left(50 \mu \mathrm{g} / \mathrm{ml}\right.$ for 30 minutes at $\left.37^{\circ} \mathrm{C}\right)$, $100 \%$ of the cells acquired the approximately 10 -second oscillations in response to LPS (Figure 7i) concomitant

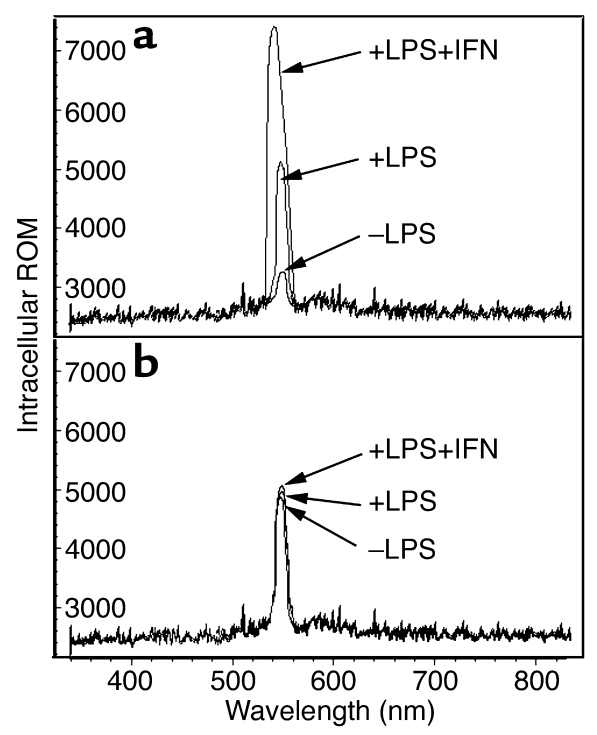

\section{Figure 9}

Intracellular ROM accumulation in neutrophils from nonpregnant (a) and pregnant (b) women. Cells were labeled with $\mathrm{H}_{2}$ TMRos. Cells were treated with buffer alone (-LPS), LPS, or LPS plus IFN- $\gamma$. The cells were quantitatively analyzed for fluorescence intensity using emission spectroscopy. ROM accumulation in cells from nonpregnant women is greatly enhanced by LPS and IFN- $\gamma(\mathbf{a})$. However, no such enhancement is seen for neutrophils from pregnant women (b). Importantly, unstimulated neutrophils from pregnant women have amounts of internal ROMs similar to those of LPS-stimulated cells from nonpregnant women. $n \geq 7$. 


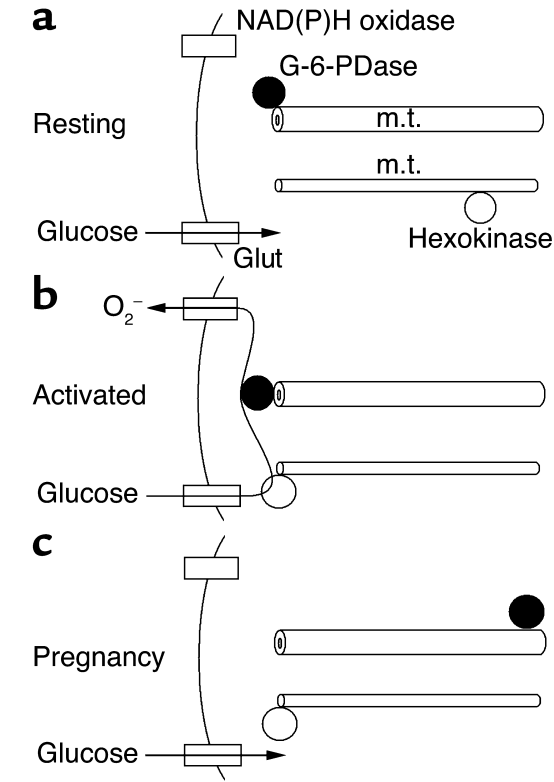

\section{Figure 10}

The potential role of metabolic enzyme trafficking in ROM release is illustrated. Conditions associated with resting $(\mathbf{a})$, activated $(\mathbf{b})$, and pregnancy $(\mathbf{c})$ neutrophils are shown. ROMs are produced during all of these conditions, but ROM production is maximal for activated cells from nonpregnant women. Hexokinase and G-6-PDase have peripheral distributions in activated cells from nonpregnant women, thus allowing maximal cooperation in ROM release. m.t., microtubule; m.f., microfilament.

with cytoplasmic randomization of G-6-PDase (Figure $4 \mathrm{n})$. The metabolic oscillations of maternal neutrophils were similarly affected by vincristine and vinblastine (data not shown; $n=3$ ). When control cells were incubated with LPS, colchicine, and 6-AN, they matched the 20 -second oscillation periods of pregnancy neutrophils (Figure 7, e and j). Hence, neutrophils of pregnant women are simultaneously primed in the sense that the metabolic oscillations are higher in amplitude, but inhibited in that they cannot acquire the approximately 10 -second metabolic period. Moreover, microtubules and the associated retrograde trafficking of G-6-PDase appear to be important in controlling metabolic flux.

Extracellular ROM release from neutrophils of pregnant and nonpregnant women. Given these alterations in G-6-PDase location and metabolism, we tested examined ROM production by neutrophils from pregnant and nonpregnant women. ROM production was monitored using $\mathrm{H}_{2}$ TMRos, which becomes fluorescent upon exposure to ROMs. To distinguish between the extracellular release of ROMs and the intracellular accumulation of ROMs, probes were incorporated into either the extracellular environment or the cellular cytoplasm using previously established methods $(35,36)$. Figure 8 shows representative ROM release experiments on neutrophils migrating in an ECM containing $\mathrm{H}_{2}$ TMRos. This single-cell ROM release assay using adherent cells in an ECM-like setting approximates the interstitial environment more closely than do cells in suspension. Furthermore, the phenotype of adherent neutrophils is distinct from that of suspended cells. For example, adherent cells produce enhanced quantities of ROMs (37). Adherent or polarized cells release low levels of ROMs (Figure 8a, trace 1) $(35,38)$. LPS, which activates the HMS and ROM release, enhanced ROM release from control neutrophils (Figure 8a, trace 2). However, LPS-mediated enhancement of ROM production is blocked by 6-AN (Figure 8a, trace 6), although 6-AN alone has no effect on basal levels of ROM release (Figure 8a, trace 4). Thus, LPS-mediated activation of ROM release from control neutrophils depends upon G-6-PDase.

The neutrophil's respiratory burst is enhanced by IFN- $\gamma(39)$. We have previously shown that IFN- $\gamma$ promotes high-amplitude $\mathrm{NAD}(\mathrm{P}) \mathrm{H}$ oscillations in adherent cells, resulting in significant levels of $\mathrm{NO}$ (34) and superoxide release (our unpublished observations). Figure $8 \mathrm{a}$, trace 3 , shows that incubation with IFN- $\gamma$ (10 $\mu \mathrm{g} / \mathrm{ml})$ for 2 hours at $37^{\circ} \mathrm{C}$ causes increased $\mathrm{ROM}$ release from adherent neutrophils. A similar response was found after PMA treatment ( $30 \mathrm{nM}$ for $30 \mathrm{~min}$ utes) (Table 1). It should be noted that, in comparing traces 2 and 3 , trace 2 rises at a higher frequency, whereas trace 3 increases with a greater step size or amplitude; both differences reflect the NAD $(\mathrm{P}) \mathrm{H}$ oscillations (Figure 7). When combined, IFN- $\gamma$ and LPS yield maximal ROM production (Figure 8a, trace 5). Further quantitative data are given in Table 1.

Figure $8 \mathrm{~b}$ demonstrates that extracellular ROM release by adherent/polarized maternal neutrophils is not increased by LPS (trace 2 ) in comparison with untreated maternal cells (trace 1). Nonetheless, the basal ROM release rate is higher in adherent pregnancy neutrophils than in neutrophils from nonpregnant women (compare Figure 8a, trace 1, with Figure 8b, trace 1). A key difference between Figure 8a and Figure $8 \mathrm{~b}$ is that the "step" frequency doubles when LPS is added to control neutrophils, but not when it is added to pregnancy neutrophils, which is consistent with the lack of 10 -secondperiod NAD $(\mathrm{P}) \mathrm{H}$ oscillations in pregnancy neutrophils (Figure $7 \mathrm{~g}$ ). The percentage of pregnancy neutrophils producing high-amplitude ROM release patterns increased from $74 \%$ to $98 \%$ after PMA treatment. In contrast to control neutrophils (Figure 8a, traces 3 and 6), ROM production by pregnancy neutrophils is not sensitive to 6-AN (Figure 8b, trace 4). Thus, the basal levels of unstimulated extracellular ROM release by adherent/ polarized cells are enhanced in pregnancy to a level comparable to those in LPS-stimulated cells from nonpregnant individuals. However, the fully activated levels brought about by incubation with LPS plus IFN- $\gamma$ in cells from nonpregnant individuals (Figure 8a, trace 5) cannot be achieved in pregnancy cells (Figure $8 b$, trace 5). LPS, IFN- $\gamma$, LPS plus IFN- $\gamma, 6$-AN, and 6-AN plus LPS did not alter ROM release from pregnancy cells in comparison with untreated cells. Hence, important changes in $\mathrm{ROM}$ production accompany pregnancy.

Microtubule-disrupting drugs rescue maximal ROM release from pregnancy neutrophils. Since colchicine normalizes 
the intracellular distribution of G-6-PDase and the LPSstimulated metabolic dynamics of pregnancy cells, we tested the ability of colchicine to normalize ROM release from maternal neutrophils. We first tested the effects of colchicine on control neutrophils. When cells from nonpregnant women were treated with colchicine $(50 \mu \mathrm{g} / \mathrm{ml}$ for 30 minutes at $37^{\circ} \mathrm{C}$ ), no changes in $\mathrm{ROM}$ production were observed relative to that in buffer-treated cells (Figure $8 c$, trace 7 vs. trace 1 ). LPS-stimulated ROM release was not significantly affected by colchicine (Figure 8c, trace 8 , vs. Figure 8 a, trace 2 ), although 6 -AN dramatically decreased ROM release from cells treated with LPS and colchicine (Figure 8c, trace 9). The higher basal rate of ROM release from pregnancy cells was not affected by colchicine (Figure 8d, traces 1 and 7). In contrast to control neutrophils, colchicine dramatically increased ROM release from LPS-stimulated pregnancy neutrophils (Figure $8 \mathrm{~d}$, trace 8 ). Enhanced ROM production is inhibited by 6 -AN (Figure $8 d$, trace 9 ). Parallel results were obtained using vincristine $(n=3)$ and vinblastine $(n=4)$, but not taxol $(n=3)$, as described above (data not shown). Thus, microtubules restrain adherent/polarized maternal neutrophils from mounting a complete oxidative program in response to activating stimuli. We suggest that G-6-PDase trafficking influences HMS activation and ROM release.

Intracellular accumulation of ROMs within neutrophils of pregnant and nonpregnant women. ROM release into the extracellular environment is not necessarily the same as intracellular ROM accumulation. To develop a more comprehensive analysis of ROM responses during pregnancy, we next evaluated the intracellular oxidation of the dye $\mathrm{H}_{2}$ TMRos in neutrophils from nonpregnant and pregnant women. $\mathrm{H}_{2}$ TMRos-labeled neutrophils were incubated with buffer alone, LPS $(50 \mathrm{ng} / \mathrm{ml}$ for $20 \mathrm{~min}$ utes), or IFN- $\gamma\left(10 \mu \mathrm{g} / \mathrm{ml}\right.$ for 2 hours at $\left.37^{\circ} \mathrm{C}\right)$ followed by addition of LPS ( $50 \mathrm{ng} / \mathrm{ml}$ for 20 minutes). Figure 9 , a and $b$, shows the fluorescence emission spectra of TMRoslabeled neutrophils from nonpregnant and pregnant women, respectively. The peak height is the total amount of TMRos formed within cells. The amount of intracellular TMRos was very small for unstimulated neutrophils from nonpregnant women but was markedly increased by the addition of LPS or of LPS plus IFN- $\gamma$ (Figure 9a). Neutrophils from pregnant women, however, did not respond with a significant change in internal ROM production. Nonetheless, the internal ROM accumulation in unstimulated cells from pregnant women was much higher than that in unstimulated neutrophils from nonpregnant women. The lack of response in maternal neutrophils was not due to saturation of the assay, since the addition of exogenous $\mathrm{H}_{2} \mathrm{O}_{2}$ caused the fluorescence level to rise dramatically (data not shown). Thus, the levels of external release and intracellular accumulation of ROM products from adherent/polarized neutrophils from pregnant women are comparable to the levels found for LPS-stimulated neutrophils from nonpregnant individuals. However, these levels cannot be enhanced by IFN- $\gamma$ as they can be in cells from nonpregnant women.

\section{Discussion}

Neutrophil activation is associated with translocation of NADPH oxidase components, granules, protein kinase $C$, and hexokinase to membranes $(23,40,41)$. We now propose a previously unknown regulatory step in neutrophil activation, retrograde translocation of G-6-PDase, which is only manifest during pregnancy. We suggest that G-6-PDase translocation is mediated by microtubules and that this redistribution accounts for diminished ROM release from maternal cells.

Several metabolic enzymes, including hexokinase, aldolase, PFK, GAPDH, and PK, bind to microfilaments and/or microtubules $(23,42-47)$. These interactions can alter both metabolic activity and cytoskeletal properties. Hexokinase translocates to the cell periphery during macrophage activation, where it increases the glucose influx rate $(22,23)$ and provides the HMS with G-6-P. The trafficking of metabolic enzymes to various cellular sites led to the concept of microcompartmentation of cell metabolism (42-47). One example of metabolic microcompartmentation is the preferential use of glycolysis as an energy source for plasma membrane functions (48-52). Although metabolic compartmentation may enhance certain cell functions, there are no unambiguous examples where different physiological states correspond to distinct compartmentation strategies. We suggest that G-6-PDase translocation in neutrophils from pregnant women constitutes a compartmentation switch regulating HMS performance.

We have identified what we believe to be a novel and fundamental difference in metabolic enzyme trafficking in neutrophils from pregnant women. Our studies have shown that G-6-PDase and G-6-PDase activity are peripherally located in control neutrophils but are located primarily in the centrosomal region of pregnancy neutrophils. This pregnancy-associated change in G-6-PDase distribution appears to be specific, as no significant differences were found for hexokinase, PFK, PK, and LDH. G-6-PDase often appeared to decorate the centrioles of stimulated cells. The apparent retrograde transport of G-6-PDase was confirmed using anti- $\gamma$-tubulin antibodies, which are specific for MTOCs (53). Colocalization and RET microscopy studies showed that G-6-PDase translocated to MTOCs in pregnancy, but not control, neutrophils.

We propose a simple model: ROM release is maximal when G-6-PDase traffics to the cell periphery, where its substrate G-6-P is readily available from hexokinase, which is also peripherally located in activated cells (Figure 10). The presence of G-6-PDase allows the HMS to be fully engaged with the local production of NADPH, the NADPH oxidase's preferred substrate. Consequently, the NADPH oxidase is provided with maximal substrate levels to yield high levels of ROM release. On the other hand, during pregnancy, G-6-PDase traffics away from the cell periphery, thereby limiting HMS efficiency by allowing glycolytic conversion of G-6-P by phosphoglucose isomerase and PFK. This is reasonable since PFK's conversion of F-6-P is irreversible and PFK maintains a 
peripheral distribution in pregnancy cells (Figure 2, $\mathrm{k}$ and 1). Perimembrane levels of NADPH would therefore be lower, making the NADPH oxidase less efficient and reducing ROM release. This hypothetical model accounts for the reduction in ROM release by maternal neutrophils. Although signal-mediated regulation of NADPH oxidase function has been extensively studied, other mechanisms may also be involved. We suggest that NADPH availability is an important factor in ROM production. We have previously proposed substrate-level control of NADPH oxidase activity (26-29). For example, the intracellular production of $\mathrm{NAD}(\mathrm{P}) \mathrm{H}$ and the extracellular release of superoxide anions oscillate in rhythm with one another with the same frequencies and phases and respond in parallel to various perturbations. The communication between these and other oscillators in activated neutrophils has been thoroughly analyzed using appropriate mathematics and computer simulations (29). The correspondence between local $\mathrm{NAD}(\mathrm{P}) \mathrm{H}$ concentration and local superoxide release from polarized neutrophils has been shown using ultrafast microscopy and microspectrophotometry (28). The present study extends our work on substratelevel control of ROM release to include G-6-PDase translocation as a means of regulating local substrate levels by metabolic microcompartmentation.

If retrograde transport of G-6-PDase affects ROM release during pregnancy, it should be possible to alter the intracellular distribution of G-6-PDase with microtubule-disrupting drugs, thereby increasing ROM production. Indeed, we have found that colchicine, vincristine, and vinblastine normalize the properties of pregnancy neutrophils. Treatment of pregnancy neutrophils with these drugs causes G-6-PDase to become more uniformly distributed, brings about the return of rapid $\mathrm{NAD}(\mathrm{P}) \mathrm{H}$ oscillations in response to LPS, and restores robust ROM release. However, the drug taxol, which has the opposite effect on microtubules, did not influence the properties of pregnancy neutrophils. This supports the idea that the intracellular distribution of G-6-PDase is an important determinant of cell metabolism and ROM production.

Our studies have shown that the HMS is intact in leukocytes from pregnant women, but that it cannot be upregulated in response to activating stimuli. In contrast, under identical conditions, cells from nonpregnant women show a roughly threefold increase in HMS activity. Parallel changes in metabolic oscillations, which represent the dynamic flux of the NAD(P)H-NADP ${ }^{+}$redox couple, were observed. Pregnancy cells demonstrated higher-amplitude oscillations than did cells of nonpregnant individuals. However, pregnancy neutrophils were unable to upregulate $\mathrm{NAD}(\mathrm{P}) \mathrm{H}$ oscillation frequency, which is closely tied to ROM production. These oscillations are likely important because (a) they encode information provided by the signal transduction apparatus, which is eventually decoded by the NADPH oxidase and NO synthase; (b) the delivery of electrons to the oxidase is compressed in time, thereby allowing short and intense bursts of activity; (c) they increase thermodynamic efficiency (54); and (d) they contribute to protecting the neutrophil from self-inflicted damage (55). Thus, pregnancy cells have HMS activity and metabolic oscillations, but neither can be upregulated in response to stimuli.

Although there are reports that superoxide release is decreased in pregnancy neutrophils (2-4), there are also reports that ROM production is increased in these cells (56). Studies reporting a decrease in ROM production measure the extracellular release of oxidants, while those reporting an increase in $\mathrm{ROM}$ production use an intracellular dye to measure ROM production. Our findings are consistent with several features of these studies, although our studies were conducted on adherent cells, not cells suspended in buffer. (The cytoskeletal reorganization associated with cell adherence may sufficiently redistribute metabolic enzymes to enhance ROM kinetics.) Our findings, like those of others (2-4), show that maternal neutrophils cannot be activated for extracellular ROM release as vigorously as neutrophils from nonpregnant individuals. We have also shown that cells from pregnant women have greater unstimulated levels of intracellular ROM accumulation than do controls. The ROM production rate of LPS-stimulated controls is similar to that of unstimulated pregnancy cells, as in other studies (56). Thus, whether pregnancy neutrophils are activated depends more on the definition of "activated" and the comparison being made than it does on the biology of the system.

The mechanism underlying retrograde transport of G-6-PDase in neutrophils during pregnancy is unknown. For example, G-6-PDase may be associated with a granule or supramolecular enzyme complex that undergoes differential translocation during nonpregnant and pregnant states. Retrograde transport of G-6-PDase apparently accounts for blunted ROM release in cells from pregnant women. This, in turn, may provide a partial explanation for the reduction in host resistance to infectious agents during pregnancy. Although retrograde G-6-PDase transport may diminish extracellular ROM release, it may enhance intracellular killing of certain microbes, since endosomes undergo retrograde transport as well. G-6-PDase translocation may affect other aspects of host defense and autoimmunity in pregnancy. For example, G-6-PDase is the rate-controlling step in ribose 5-phosphate synthesis, which is required for cell proliferation (57). Thus, the mechanism proposed might be more broadly involved in the control of immune responses. We are presently studying the mechanisms regulating G-6-PDase trafficking, with the long-range goal of improving disease management.

\section{Acknowledgments}

This research was supported by the National Institute of Allergy and Infectious Diseases and the National Multiple Sclerosis Society (to H.R. Petty) and by the National Institute of Child Health and Human Development (to R. Romero). 
1. Buyon, J.P. 1998. The effects of pregnancy on autoimmune diseases. J. Leukoc. Biol. 63:281-287.

2. Crouch, S.P.M., Crocker, I.P., and Fletcher, J. 1995. The effect of pregnancy on polymorphonuclear leukocyte function. J. Immunol. 155:5436-5443.

3. Cotton, D.J., Seligmann, B., O’Brian, B., and Gallin, J.I. 1983. Selective defect in human neutrophil superoxide anion generation elicited by the chemoattractant $\mathrm{N}$-formylmethionylleucylphenylalanine in pregnancy. J. Infect. Dis. 148:194-199.

4. Tsukimori, K., et al. 1993. The superoxide generation of neutrophils in normal and preeclamptic pregnancies. Obstet. Gynecol. 81:536-540.

5. Benjamin, J.L., and Remington, J.S. 1984. The adverse effect of pregnancy on macrophage activation. Cell. Immunol. 85:94-99.

6. El-Maallem, H., and Fletcher, J. 1980. Impaired neutrophil function and myeloperoxidase deficiency in pregnancy. Br. J. Haematol. 44:375-381.

7. Krause, P.J., et al. 1987. Host defense during pregnancy: neutrophil chemotaxis and adherence. Am. J. Obstet. Gynecol. 157:274-280.

8. Persellin, R.H., and Thoi, L.L. 1979. Human polymorphonuclear leukocyte phagocytosis in pregnancy: development of inhibition during gestation and recovery in the post partum period. Am. J. Obstet. Gynecol. 134:250-255.

9. Brabin, B.J. 1985. Epidemiology of infection in pregnancy. Rev. Infect. Dis. 7:579-603

10. Larsen, B., and Galask, R.P. 1978. Host-parasite interactions during pregnancy. Obstet. Gynecol. Surv. 33:297-318.

11. Luft, B.J., and Remington, J.S. 1982. Effect of pregnancy on resistance to Listeria monocytogenes and Toxoplasma gondii infections in mice. Infect. Immun. 38:1164-1171.

12. Cunningham, F.G., Leveno, K.J., Hankins, G.D.V., and Whalley, P.J. 1984. Respiratory insufficiency associated with pyelonephritis during pregnancy. Obstet. Gynecol. 63:121-125.

13. Malech, H.L., and Gallin, J.I. 1987. Neutrophils in human diseases. N. Engl. J. Med. 317:687-694.

14. Confavreux, C., Hutchinson, M., Hours, M.M., Cortinovis-Tourniaire, P., and Moreau, T. 1998. Rate of pregnancy-related relapse in multiple sclerosis. N. Engl. J. Med. 339:285-291.

15. Spector, T.D., and da Silva, J.A.P. 1992. Pregnancy and rheumatoid arthritis. Clin. Rheumatol. 11:189-194.

16. Agarwal, R.K., Chan, C.-C., Wiggert, B., and Caspi, R.R. 1999. Pregnancy ameliorates induction and expression of experimental autoimmune uveitis. J. Immunol. 162:2648-2654.

17. Chanock, S.J., el Benna, J., Smith, R.M., and Babior, B.M. 1994. The respiratory burst oxidase. J. Biol. Chem. 269:24519-24522.

18. Tan, A.S., Ahmed, N., and Berridge, M.V. 1998. Acute regulation of glucose transport after activation of human peripheral blood neutrophils by phorbol myristate acetate, fMLP, and granulocyte-macrophage colonystimulation factor. Blood. 91:649-655.

19. Ozcan, S., and Johnston, M. 2000. Function and regulation of yeast hexose transporters. Microbiol. Mol. Biol. Rev. 63:554-569.

20. Rist, R.J., Jones, G.E., and Naftalin, R.J. 1991. Effects of macrophage colony-stimulating factor and phorbol myristate acetate on 2-Ddeoxyglucose transport and superoxide production in rat peritoneal macrophages. Biochem. J. 278:119-128.

21. Naftalin, R.J., and Rist, R.J. 1993. The relationship between sugar metabolism, transport and superoxide radical production in rat peritoneal macrophages. Biochim. Biophys. Acta. 1148:39-50.

22. Kiyotaki, C., Peisach, J., and Bloom, B.R. 1984. Oxygen metabolism in cloned macrophage cell lines: glucose dependence of superoxide production, metabolic and spectral analysis. J. Immunol. 132:857-866.

23. Pedley, K.C., Jones, G.E., Magnani, M., Rist, R.J., and Naftalin, R.J. 1993. Direct observation of hexokinase translocation in stimulated macrophages. Biochem. J. 291:515-522.

24. Bender, J.G., and van Epps, D.E. 1985. Inhibition of human neutrophil function by 6 -aminonicotinamide: the role of the hexose monophosphate shunt in cell activation. Immunopharmacology. 10:191-199.

25. Roos, D., and Balm, A.J.M. 1980. The oxidative metabolism of monocytes. In The reticuloendothelial system: a comprehensive treatise. A.J. Sbarra and R.R Strauss, editors. Plenum Press. New York, New York, USA. 198-229.

26. Petty, H.R. 2001. Neutrophil oscillations: temporal and spatiotemporal aspects of cell behavior. Immunol. Res. 23:125-134.

27. Petty, H.R., and Kindzelskii, A.L. 2001. Dissipative metabolic patterns respond during neutrophil transmembrane signaling. Proc. Natl. Acad. Sci. USA. 98:3145-3149.

28. Kindzelskii, A.L., and Petty, H.R. 2002. Apparent role of traveling metabolic waves in periodic oxidant release by living cells. Proc. Natl. Acad. Sci. USA. 99:9207-9212.

29. Olsen, L.F., Kummer, U., Kindzelskii, A.L., and Petty, H.R. 2003. A model of the oscillatory metabolism of activated neutrophils. Biophys. J. In press.

30. Kindzelskii, A.L., Eszes, M.M., Todd, R.F., III, and Petty, H.R. 1997. Proximity oscillations of complement receptor type 4 and urokinase receptors on migrating neutrophils are linked with signal transduction/metabolic oscillations. Biophys. J. 73:1777-1784.
31. DeChatelet, L.R., and Parce, J.W. 1981. Hexose monophosphate shunt activity and oxygen uptake. In Methods for studying mononuclear phagocytes. D.O. Adams, P.J. Edelson, and H. Koren, editors. Academic Press Inc. New York, New York, USA. 477-488.

32. Babior, B.M., and Cohen, H.J. 1981. Measurement of neutrophil function: phagocytosis, degranulation, the respiratory burst and bacterial killing. In Methods in hematology: leukocyte function. M.J. Cline, editor. Churchill Livingstone. New York, New York, USA. 1-39.

33. Butcher, R.G., and Van Noorden, C.J.F. 1985. Reaction rate studies of glucose-6-phosphate dehydrogenase activity in section of rat liver using four tetrazolium salts. Histochem. J. 17:993-1008.

34. Adachi, Y., Kindzelskii, A.L., Ohno, N., Yadomae, T., and Petty, H.R. 1999. Amplitude and frequency modulation of metabolic signals in leukocytes: synergistic role in interferon- $\gamma$ and interleukin-6-mediated cell activation. J. Immunol. 163:4367-4374.

35. Kindzelskii, A.L., Zhou, M.-J., Haugland, R.P., and Petty, H.R. 1998. Oscillatory pericellular proteolysis and oxidant deposition during neutrophil migration. Biophys. J. 74:90-97.

36. Cao, D., Boxer, L.A., and Petty, H.R. 1993. Deposition of reactive oxygen metabolites onto and within living tumor cells during neutrophil-mediated antibody-dependent cellular cytotoxicity. J. Cell. Physiol. 156:428-436

37. Nathan, C.F. 1987. Neutrophil activation on biological surfaces: massive secretion of hydrogen peroxide in response to products of macrophages and lymphocytes. J. Clin. Invest. 80:1550-1560.

38. Demaurex, N., Downey, G.P., Waddell, T.K., and Grinstein, S. 1996. Intracellular $\mathrm{pH}$ regulation during spreading of human neutrophils. J. Cell Biol. 133:1391-1402.

39. Berton, G., Zeni, L., Cassatella, M.A., and Rossi, F. 1986. Gamma interferon is able to enhance the oxidative metabolism of human neutrophils. Biochem. Biophys. Res. Commun. 138:1276-1282.

40. Nixon, J.B., and McPhail, L.C. 1999. Protein kinase C (PKC) isoforms translocate to triton-insoluble fractions in stimulated human neutrophils: correlation of conventional PKC with activation of NADPH oxidase. J. Immunol. 163:4574-4582.

41. Price, M.O., et al. 2002. Creation of a genetic system for analysis of the phagocyte respiratory burst: high level reconstitution of the NADPH oxidase in a nonhematopoietic system. Blood. 99:2653-2661.

42. Cortassa, S., Caceres, A., and Aon, M.A. 1994. Microtubular protein in its polymerized or nonpolymerized states differentially modulates in vitro and intracellular fluxes catalyzed by enzymes of carbon metabolism. J. Cell. Biochem. 55:120-132.

43. Vertessay, B.G., Orosz, F., Kovacs, J., and Ovadi, J. 1997. Alternative binding of two sequential glycolytic enzymes to microtubules. J. Biol. Chem 272:25542-25546.

44. Minaschek, G., Groschel-Steward, U., Blum, S., and Bereiter-Hahn, J. 1992. Micro-compartmentation of glycolytic enzymes in cultured cells. Eur. J. Cell Biol. 58:418-428.

45. Pagliaro, L., and Taylor, D.L. 1992. 2-Deoxyglucoase and cytochalasin D modulate aldolase mobility in living 3 T3 cells. J. Cell Biol. 118:859-863.

46. Roberts, S.J., and Somero, G.N. 1987. Binding of phosphofructokinase to filamentous actin. Biochemistry. 26:3437-3442.

47. Wang, J., Tolan, D.R., and Pagliaro, L. 1997. Metabolic compartmentation in living cells: structural association with aldolase. Exp. Cell Res. 237:445-451

48. Weiss, J.N., and Korge, P. 2001. The cytoplasm: no longer a well-mixed bag. Circ. Res. 89:108-110.

49. Weiss, J.N., and Lamp, S.T. 1987. Glycolysis preferentially inhibits ATPsensitive $\mathrm{K}+$ channels in isolated guinea pig cardiac myocytes. Science. 238:67-69.

50. Glitsch, H.G., and Tappe, A. 1993. The $\mathrm{Na}^{+} / \mathrm{K}+$ pump of cardiac Purkinje cells is preferentially fueled by glycolytic ATP production. Pflugers Arch. 422:380-385

51. Kennedy, H.J., et al. 1999. Glucose generates sub-plasma membrane ATP microdomains in single islet $\beta$-cells. J. Biol. Chem. 274:13281-13291.

52. Mertz, R.J., Worley, J.F., Spencer, B., Johnson, J.H., and Dukes, I.D. 1996. Activation of stimulus-secretion coupling in pancreatic $\beta$-cells by specific products of glucose metabolism. J. Biol. Chem. 271:4838-4845.

53. Jeng, R., and Stearns, T. 1999. $\gamma$-Tubulin complexes: size does matter. Trends Cell Biol. 9:339-342.

54. Lazar, J.G., and Ross, J. 1990. Changes in mean concentration, phase shifts, and dissipation in a forced oscillatory reaction. Science. 247:189-192.

55. Hauser, M.J.B., Kummer, U., Larsen, A.Z., and Olsen, L.F. 2001. Oscillatory dynamics protect enzymes and possibly cells against toxic substances. Faraday Discuss. 120:215-227.

56. Sacks, G.P., Studena, K., Sargent, I.L., and Redman, C.W.G. 1998. Normal pregnancy and preeclampsia both produce inflammatory changes in peripheral blood leukocytes akin to those of sepsis. Am. J. Obstet. Gynecol. 179:80-86

57. Tian, W.N., et al. 1998. Importance of glucose-6-phosphate dehydrogenase activity for cell growth. J. Biol. Chem. 273:10609-10617. 